\title{
A magnetically actuated ball valve applicable for small-scale fluid flows
}

\section{Smistrup, Kristian; Stone, Howard A.}

Published in:

Physics of Fluids

Link to article, DOI:

$10.1063 / 1.2717690$

Publication date:

2007

Document Version

Publisher's PDF, also known as Version of record

Link back to DTU Orbit

Citation (APA):

Smistrup, K., \& Stone, H. A. (2007). A magnetically actuated ball valve applicable for small-scale fluid flows. Physics of Fluids, 19(6), 063101. https://doi.org/10.1063/1.2717690

\section{General rights}

Copyright and moral rights for the publications made accessible in the public portal are retained by the authors and/or other copyright owners and it is a condition of accessing publications that users recognise and abide by the legal requirements associated with these rights.

- Users may download and print one copy of any publication from the public portal for the purpose of private study or research.

- You may not further distribute the material or use it for any profit-making activity or commercial gain

- You may freely distribute the URL identifying the publication in the public portal

If you believe that this document breaches copyright please contact us providing details, and we will remove access to the work immediately and investigate your claim. 


\title{
A magnetically actuated ball valve applicable for small-scale fluid flows
}

Kristian Smistrup

Division of Engineering and Applied Sciences, Harvard University, Cambridge, Massachusetts 02138

and MIC, Department of Micro and Nanotechnology, Technical University of Denmark,

Building 345 East, DK-2800 Kongens Lyngby, Denmark

Howard A. Stone ${ }^{\text {a) }}$

Division of Engineering and Applied Sciences, Harvard University, Cambridge, Massachusetts 02138

(Received 4 September 2006; accepted 9 January 2007; published online 27 June 2007)

\begin{abstract}
We present three possible designs for magnetically actuated ball valves that can be scaled down to nanometer length scales. Analytical expressions are presented for the hydraulic resistance of the ball valve as a function of geometric parameters and the state of the valve, and we also present analytical expressions for the hydrodynamic force on the magnetic bead that functions as the ball in the valve. We verify these expressions numerically and calculate the magnetic forces that can be exerted on the magnetic bead using the proposed structures. Finally, for typical parameters we show that these structures will be able to withstand a back pressure between 3 and $30 \mathrm{kPa}$ regardless of the size of the bead/ball. (c) 2007 American Institute of Physics. [DOI: 10.1063/1.2717690]
\end{abstract}

\section{INTRODUCTION}

The spectrum of research and development activities that are impacted by microfluidic devices continues to grow at a rapid pace. ${ }^{1,2}$ One significant reason for the growth is the ability to control fluid flow and chemical composition at the scale of tens and hundreds of microns. Future developments will likely occur in many areas of biology, chemistry, and engineering, such as fields requiring increased precision or control, and where scaling down to smaller (e.g., nanoscales) brings additional advantages. The opportunity to control fluid flows without the need of external mechanical linkages provides one pathway for innovation.

Lab on a chip systems are being developed for use in chemical analysis, synthesis, identification, and delivery. ${ }^{3,4}$ In many of these systems it is problematic to make small valves. This difficulty will only be accentuated as designs are scaled down. As one illustration, in recent years systems have been proposed for timed drug delivery. ${ }^{5,6}$ For example, a diabetes patient needs to take doses of insulin at given time intervals. Imagine a system with $10^{5}$ small compartments filled with insulin. If each of these compartments could be opened or closed by a magnetic valve, it would be possible to do timed delivery to the patient by integrating a small chip into the patients body. Of course, there are other routes to timed drug delivery.

Conventional valves in microsystems are commonly used for fluid handling and in micropumps. They can be divided into two main classes: Passive valves that basically function as fluid rectifiers blocking the flow in one direction, but not the other, and active valves, where some form of actuation is used to open or close the valve. Pneumatic, thermopneumatic, electrostatic, piezoelectric, or electromagnetic actuation is commonly used. ${ }^{7-9}$ Almost all of these designs are fabricated using silicon micromachining and MEMS

$\overline{{ }^{a} \text { Electronic mail: has@deas.harvard.edu }}$ technology, and almost all of them involve a silicon or rubber-like diaphragm or a silicon-based cantilever. In general these valve designs can withstand pressures of a few $\mathrm{kPa}$. Also, a few (passive) ball valve designs exist. ${ }^{10,11}$

In addition, a few polymer valve designs have been demonstrated. Simple twist valves were used with soft lithography, ${ }^{12}$ and a PDMS soft-lithography design of an active pneumatic valve was demonstrated. ${ }^{13}$ Other active and passive valve designs include a polymer plug moving inside a glass microfluidic system. ${ }^{14-16}$ These designs have proven effective at pressures greater than $30 \mathrm{MPa}^{16}$

The above active valve types do not scale down easily, as the designs require relatively large structures for actuation, and/or moving parts. A nanoscale cantilever based design was presented recently. ${ }^{17}$ To the best of the authors' knowledge the only experimentally realized nanoscale valve design is a chemically actuated molecular valve. ${ }^{18}$

In this paper we propose an active valve arrangement using a magnetic bead or particle, which is actuated magnetically, and is suitable for the regulation of small-scale fluid flows. The necessary magnetic forces are created by passive elements of soft magnetic material that create strong magnetic field gradients, when they are magnetized by an external magnetic field. Magnetic beads and particles are available with a range of magnetic properties and sizes ranging from $10 \mu \mathrm{m}$ down to $5 \mathrm{~nm} .{ }^{19}$ We believe that our design is realizable in both PDMS and silicon, and can be scaled down to the nanoscale. We calculate analytically using a lubrication approach (and verify numerically) the flow in our valve arrangement. Furthermore, we present scaling arguments and demonstrate numerically that the proposed geometry will produce sufficient magnetic force to operate the valves at reasonable pressures and flow rates. At these length scales Brownian motion plays an important role. We do not include Brownian motion directly in the following, but at the end we discuss the possible influence of this random motion on the results. 

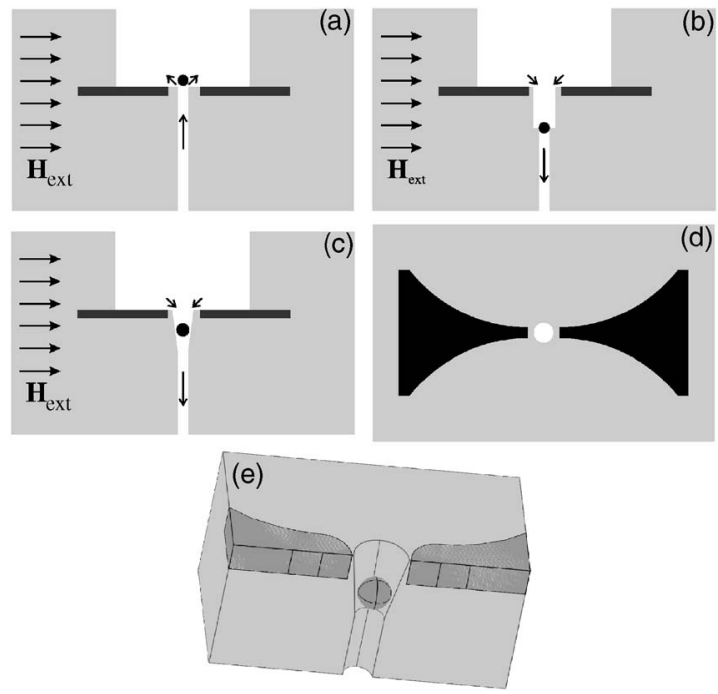

FIG. 1. (a)-(c) Cross sections of the three proposed geometries for magnetically actuated ball valves. The dark gray "plates" are slabs of soft magnetic material. The lighter gray is the silicon or PMDS structure that defines the microfluidic network. The solid black circle is the magnetic bead or particle. The arrows indicate the expected direction of the fluid flow and the external magnetic field, $\mathbf{H}_{\mathrm{ext}}$. (a) In this configuration the magnetic field is constantly on, and thus the magnetic bead blocks the outlet of a channel. (b) An alternative configuration, where the magnetic field is initially off, but the fluid pressure holds the bead in the fluid inlet which is thus blocked. Turning on the magnetic field lifts the bead out of the inlet. (c) Same principle as (b), but the design has tapering sidewalls. This makes it easier to control the bead position, but it may be harder to fabricate than (b). (d) The magnetic structure viewed from above as well as the position of the fluid channel. It is seen how only the fluid channel has cylindrical symmetry. (e) 3D image of a spherical bead in the opening of the tapering tube. The dark gray is the magnetic material, the light gray is the silicon/PDMS, and the spherical object is the bead.

Figure 1 shows the proposed geometries for small-scale externally actuated valves. In all the designs the small fluid channel has a circular cross section, whereas the large channel or reservoir can have any shape; generalization to other shapes is, of course, possible. Figures 1(a) and 1(b) both have a small channel with straight sidewalls. In Fig. 1(a) the bead is envisioned to be smaller than the channel opening, and thus fluid flow is regulated (but not blocked totally) by controlling the position of the bead. The design of Fig. 1(a) also works well if the bead is larger than the channel opening, but in that case one might choose the design of Fig. 1(b), where the fluid pressure forces the bead to block the flow. By applying a magnetic force the valve can be opened. The structure in Fig. 1(c) has tapering sidewalls, and thus the bead will block the flow at some position in the tapering channel. This design has the advantage that the bead is forced into the opening by the fluid pressure, and also the bead diameter is less critical.

The thin magnetic slabs are shown from above in Fig. 1(d) and from the side in Figs. 1(a)-1(c) and Fig. 1(e). For this type of external magnetic actuation we imagine that the slabs are composed of a soft magnetic material, e.g., permalloy or CoNiFe. When these materials are magnetized by an external magnetic field, strong magnetic fields and field
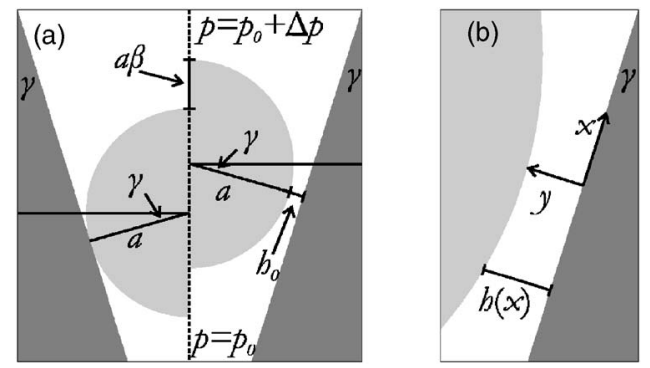

FIG. 2. (a) The calculation geometry for the tapered tube configuration of Fig. 1(c). A bead with radius $a$ is inserted into a tapering tube. There is cylindrical symmetry around the dashed line. The situation where the bead is resting in the tube is shown on the left-hand side of the dashed line, and in this situation there can be no flow. On the right-hand side of the dashed line is shown the bead lifted a distance $\beta a$ from the resting situation, and in this case, there is fluid flow. The inclination angle of the tube is $\gamma$, and the pressure drop across the sphere is $\Delta p$. (b) Definition of the coordinate system used. $h(x=0)=h_{0}=a \beta \sin \gamma$.

gradients are created in the vicinity of the gap (fluid inlet/ outlet). These fields exert a force on the bead, which pulls the bead out of or into the fluid inlet/outlet, and so regulates fluid flow.

\section{THEORY}

In this section, we set up the general equations for calculations of the fluid flow in the narrow gaps around the magnetic bead. We will show in detail how the problem in Fig. 1(c) is solved, and we will give the final results for the other configurations. We will also give a scaling argument for the magnetic force that shows that it is indeed possible to create sufficient force to manipulate the bead for regulation of the flow.

\section{A. Analytical solution for the tapered tube}

Figure 2 introduces the flow geometry for the tapered tube version of the valve. Two geometric parameters characterize the valve configuration: (i) $\beta$ is the vertical displacement of the sphere center from a (no flow) resting position against the channel walls, and (ii) $\gamma$ is the inclination angle of the taper. We study the flow problem in the lubrication limit, where the gap between the tube wall and the bead has minimum size, $h_{0}=a \beta \sin \gamma$, and where the gap is narrow, i.e., $h_{0} \ll a$. The geometry is rotationally symmetric around the dashed line in Fig. 2(a), but since $h_{0} \ll a$, the coordinate system can be viewed locally as Cartesian, so we use the Cartesian coordinate system indicated in Fig. 2(b).

In the lubrication limit, everything is determined by the flow in the narrow gap, where we can approximate the shape of the spherical bead by a parabolic shape and obtain

$$
h(x)=h_{0}\left(1+\frac{x^{2}}{2 h_{0} a}+\mathcal{O}\left(\frac{x^{4}}{h_{0} a^{3}}\right)\right) .
$$

This shape function establishes the characteristic length scale, $x_{0}=\sqrt{2 h_{0} a}$. Since everything is determined by the flow in the gap, only the part, $|x|<x_{0}$, matters, and thus $h(x)$ $=h_{0}\left(1+\frac{x^{2}}{2 h_{0} a}\right)$ will suffice as an approximation to the shape. 
With these opening statements the derivation of the flow, $\mathbf{u}=u(x, y) \hat{\mathbf{e}}_{x}+v(x, y) \hat{\mathbf{e}}_{y}$, and pressure distribution, $p(x, y)$, follows the usual path of lubrication analysis, where we consider the lubrication equations $\mathrm{s}^{20,21}$

$$
\begin{aligned}
& \frac{\partial p}{\partial x}=\eta \frac{\partial^{2} u}{\partial y^{2}}, \\
& \frac{\partial p}{\partial y}=0, \\
& \frac{\partial u}{\partial x}+\frac{\partial v}{\partial y}=0 .
\end{aligned}
$$

Equation (2b) states that the pressure does not depend on $y$, and therefore Eq. (2a) can be integrated to give

$$
u(x, y)=\frac{1}{2 \eta} \frac{\partial p}{\partial x} y(h(x)-y),
$$

where no-slip boundary conditions have been imposed at $y=0$ and $y=h(x)$.

In order to find the pressure gradient, we integrate over a cross section of the fluid domain to obtain the total flow rate $Q$ at any $x$,

$$
Q=2 \pi a \sin \gamma \int_{0}^{h(x)} u(x, y) d y=\frac{\pi a \sin \gamma}{6 \eta} \frac{\partial p}{\partial x} h^{3}(x) .
$$

Continuity requires that $Q$ must be independent of $x$, so Eq. (4) relates the flow rate, the position, and the pressure gradient in a unique way. The total flow rate is determined by integrating Eq. (4),

$$
\Delta p=\frac{6 \eta Q}{\pi a \sin \gamma} \int_{-\infty}^{\infty}(h(x))^{-3} d x=\frac{9 \eta Q \csc \gamma}{2 \sqrt{2 a h_{0}^{5}}},
$$

where $\Delta p$ is the pressure drop across the sphere.

Combining Eqs. (3)-(5) thus determines the flow and pressure fields, while the hydrodynamic resistance is

$$
\frac{\eta Q}{a^{3} \Delta p}=\frac{4}{9 \sqrt{2}} \beta^{5 / 2}(\sin \gamma)^{7 / 2},
$$

which depends on the two geometric parameters $\beta$ and $\gamma$.

To obtain the hydrodynamic force on the sphere, we note that in the lubrication limit the force is dominated by the pressure force. ${ }^{20}$ Thus we obtain the hydrodynamic force in the opposite direction of the pressure gradient,

$$
F^{h}=\pi(a \cos \gamma)^{2} \Delta p .
$$

Note that if we consider the force and flow rate specified, then, for a given inclination angle $\gamma$, the position $\beta$ of the sphere is determined.

\section{B. Straight channel geometry}

Figure 3 defines the calculation geometry for the flow calculations for Figs. 1(a) and 1(b). Again there are two geometric parameters, (i) the difference $\varepsilon a$ between the radius of
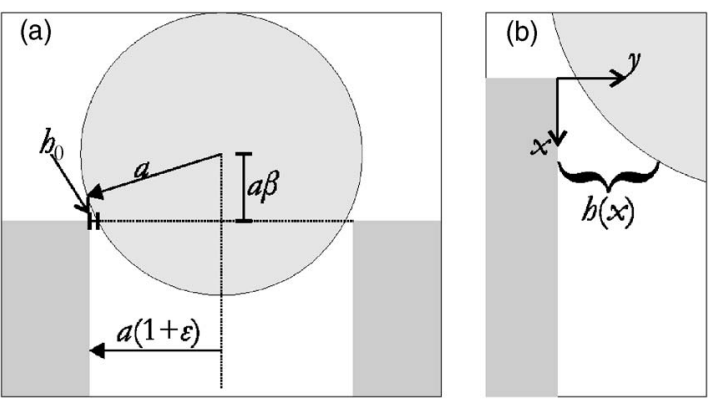

FIG. 3. (a) Definition of variables for the geometry where the channel is straight, i.e., Figs. 1(a) and 1(b). A bead with radius $a$ is positioned at the opening of a tube (marked by the dark gray) with radius $a(1+\varepsilon)$. If $\varepsilon>0$, we have the situation in Fig. 1(a) and if $\varepsilon<0$, we have the situation in Fig. 1(b). $\varepsilon<0$ is shown here. The vertical distance between the center of the bead and the opening of the tube is $a \beta$. The pressure drop across the sphere is $\Delta p$. (b) Definition of the coordinate system.

the feed channel and the sphere radius, and (ii) the vertical displacement $\beta a$ of the bead. If $\varepsilon>0$, we have Fig. 1(a) and when $\varepsilon<0$, we have Fig. 1(b).

In both cases, a little geometry gives us the height as $h(x)=a\left(\varepsilon+\beta^{2} / 2\right)+\beta x+x^{2} /(2 a)$, which can be rewritten as

$$
h(x)=h_{0}\left(1+\frac{2 \beta}{\sqrt{\beta^{2}+2 \varepsilon}} \frac{x}{\sqrt{2 a h_{0}}}+\frac{x^{2}}{2 a h_{0}}\right),
$$

where $h_{0}=h(0)=a\left(\varepsilon+\beta^{2} / 2\right)$ is the minimum height in the gap, and $a, \varepsilon$, and $\beta$ were defined in Fig. 3. We notice that for $\varepsilon<0$, we must require that $\beta>\beta_{\min } \equiv \sqrt{-2 \varepsilon}$.

Following the steps of Eqs. (3)-(5), we arrive at

$$
\Delta p=\frac{6 \eta Q}{\pi a} \int_{0}^{\infty}(h(x))^{-3} d x,
$$

which immediately gives

$$
\begin{aligned}
\frac{\eta Q}{a^{3} \Delta p}= & \frac{\pi( \pm \varepsilon)^{5 / 2}}{48}\left(\lambda^{2} \pm 2\right)^{5 / 2} \\
& \times\left[\int_{0}^{\infty}\left(1+\frac{2 \lambda}{\sqrt{\lambda^{2} \pm 2}} s+s^{2}\right)^{-3} d s\right]^{-1},
\end{aligned}
$$

where $\lambda=\beta( \pm \varepsilon)^{-1 / 2}$, and the plus signs are used, if $\varepsilon>0$, and the minus signs are used for $\varepsilon<0$. The force on the bead is $F^{h}=\pi a^{2} \Delta p$, if $\varepsilon>0$ and $F^{h}=\pi a^{2}(1+\varepsilon)^{2} \Delta p$ otherwise.

Since the requirement $\beta>\beta_{\min }$ ensures that the integrand is always real, the integral in Eq. (10) is easily evaluated numerically, and a closed form expression can also be given, but this form is messy and does not convey any new information.

Finally, we show how this result behaves in different limits,

$$
\begin{aligned}
& \frac{\eta Q}{a^{3} \Delta p}=\frac{5}{48} \pi \beta^{5}, \quad \varepsilon=0, \\
& \frac{\eta Q}{a^{3} \Delta p}=\frac{4}{9} \sqrt{2} \varepsilon^{5 / 2}, \quad \varepsilon>0, \beta \rightarrow 0,
\end{aligned}
$$




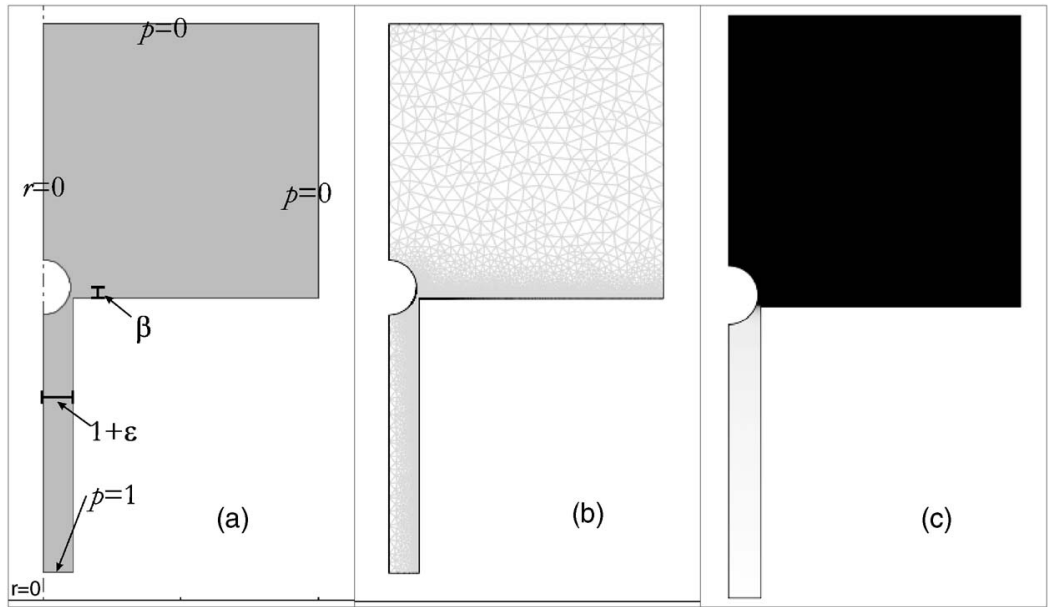

FIG. 4. Example of a Comsol Multiphysics calculation. (a) The calculation geometry with all lengths scaled by the sphere radius. The magnetic bead is treated as a stationary sphere with no-slip boundary conditions on the surface. The boundary conditions for the dimensionless pressure distribution and fluid flow are shown on the figure; if the boundary condition is not explicitly shown, no-slip conditions have been imposed. The dimensionless geometric parameters $\varepsilon$ and $\beta$ are defined. (b) An example of the mesh used for the numerical calculation. The mesh is very fine in the gap region, where most of the interesting fluid dynamics take place. (c) An example of a numerical result for small values of $\beta>|\varepsilon|$. The gray scale surface plot shows the pressure field, where since the change from black to white occurs abruptly across the gap region; the entire pressure drop occurs over the narrow gap.

$$
\frac{\eta Q}{a^{3} \Delta p}=\frac{\pi}{3} \beta_{\min }^{3}\left(\beta-\beta_{\min }\right)^{2}, \quad \varepsilon<0, \beta \rightarrow \beta_{\min } .
$$

\section{Magnetic force scaling law}

The magnetic force on a magnetic particle is ${ }^{22}$

$$
\mathbf{F}^{\mathrm{mag}}=\mu_{0} \int_{V_{\text {bead }}}(\mathbf{M} \cdot \boldsymbol{\nabla}) \mathbf{H}_{\mathrm{ext}} d v,
$$

where $\mu_{0}$ is the magnetic permeability of free space, $\mathbf{M}$ is the magnetization of the bead, $\mathbf{H}_{\mathrm{ext}}$ is the magnetic field that would be present, if the bead were not, and $V_{\text {bead }}$ is the volume of the bead.

The magnetization of the bead is limited by the saturation magnetization of the bead, $M_{s, \text { bead }}$, and since an applied homogenous field will not contribute to the force, only the field disturbance caused by the magnetic slabs [shown in Fig. 1(d)] will contribute to the gradient. This disturbance is limited by the saturation magnetization of the soft magnetic material, $M_{s, \text { slab. }}$. The characteristic length scale of the magnetic gradient outside the gap is set by a combination of the thickness of the two magnetic slabs and the distance between their tips. In order to achieve the largest gradients this distance should be as small as possible, and therefore it should also scale with the bead diameter, and so the length scale for the magnetic field gradient is the bead diameter. Combined, these statements say that for a sufficiently large applied magnetic field, the force that pulls the particle towards the gap between the magnetic plates is approximately

$$
\begin{aligned}
F^{\mathrm{mag}} & =\mathcal{O}\left(\frac{4}{3} \pi a^{3} \mu_{0} M_{s, \text { bead }} \frac{M_{s, \text { slab }}}{2 a}\right) \\
& =\mathcal{O}\left(2 a^{2} \mu_{0} M_{s, \text { bead }} M_{s, \text { slab }}\right) .
\end{aligned}
$$

For a $\mathrm{MyOne}^{23}$ magnetic bead from Invitrogen $\left(2 a=1.05 \mu \mathrm{m}, M_{s, \text { bead }} \approx 40 \mathrm{kA} / \mathrm{m}\right)$ and permalloy (Py) magnetic slabs $\left(M_{s, \mathrm{Py}} \approx 8.8 \cdot 10^{5} \mathrm{~A} / \mathrm{m}\right)$ this force is approximately $24 \mathrm{nN}$.

If we compare Eqs. (7) and (13), we obtain an estimate for the maximum applied pressure for a given flow configuration,

$$
\Delta p_{\max }=\mathcal{O}\left(\frac{2}{\pi \cos ^{2} \gamma} \mu_{0} M_{s, \text { bead }} M_{s, \text { slab }}\right),
$$

which is independent of the diameter of the particle and is approximately $30 \mathrm{kPa}=0.3$ bar for a small angle $(\gamma$; Fig. 2) and the aforementioned parameters.

\section{NUMERICAL APPROACH AND RESULTS}

We have performed numerical calculations for the fluid flow [for the configurations in Figs. 1(a) and 1(b)] and magnetic field distribution [for the configuration in Fig. 1(d)] using Comsol Multiphysics 3.2a. The calculations allow us to explore configurations beyond the narrow gap limit considered in Sec. II.

\section{A. Numerical methods for the fluid motion}

The calculations for the fluid flow were performed in a two-dimensional cylindrical coordinate system $(r, z)$ with rotational symmetry around the $z$ axis. Figure 4 shows the calculation geometry, an example of a mesh, and an example of the result from this kind of calculation.

Since we expect this valve arrangement to be applied on the microscale, and since the fluid dynamics is dominated by the small gap between the bead and the outlet of the tube, we neglect the inertial term in the Navier-Stokes equations, and thus we only solve the Stokes equations combined with the continuity equation. We lose no generality by using dimensionless variables, so in the calculations we have the dimen- 
sionless position, $\tilde{\mathbf{x}}=\mathbf{x} / a$, where $a$ is the bead radius, fluid velocity, $\tilde{\mathbf{u}}=\mathbf{u} \eta /(a \Delta p)$, and pressure, $\tilde{p}=\left(p-p_{0}\right) / \Delta p$, where $\Delta p$ is the applied pressure drop and $p_{0}$ is the ambient pressure.

For completeness we note that the meshes were set up by choosing the "Finer" mesh setting under Mesh Parameters, except that the "Resolution of narrow regions" parameter was set to 3, and the "Maximum element size" and the "Element growth rate" was set to $10^{-4}$ and 1.007 , respectively, at the corner of the cylindrical tube closest to the bead. Figure 4(b) shows an example of such a mesh.

A sample result of this type of calculation is shown in Fig. 4(c). The dimensionless pressure field is shown as a gray scale surface plot. The white regions have high pressure, $\tilde{p}$ $\approx 1$ and the black regions have low pressure, $\tilde{p} \approx 0$. It is observed that the entire pressure drop happens across the small gap between the bead and the end of the cylindrical tube.

We have extracted the total flow rate from the results using Comsol Multiphysics' built-in postprocessing integration routine. The flow rate is obtained by integrating $Q=\int 2 \pi r v_{z} d r$ along the boundary where $p=1$. The force on the bead is calculated by integration of the total stress tensor over the surface of the bead.

\section{B. Numerical method for the magnetic force calculations}

The magnetic calculations were performed using the Electromagnetics module in Comsol Multiphysics. We are considering a situation without currents, and thus the $\mathbf{H}$ field is curl free and can be written as the gradient of a scalar potential, $\mathbf{H}=-\boldsymbol{\nabla} \psi$. The potential can be found as the solution to $\boldsymbol{\nabla} \cdot \mathbf{B}=-\boldsymbol{\nabla} \cdot\left(\mu_{0} \mu_{r}(|\mathbf{H}|) \nabla \psi\right)=0$, where $\mathbf{B}$ is the magnetic flux density, $\mu_{0}$ is the permeability of free space, and $\mu_{r}(|\mathbf{H}|)$ is the relative permeability that depends on the magnitude of the $\mathbf{H}$ field. In general $\mu_{r}$ is a nonlinear hysteretic tensor, but in these calculations, it is treated as a nonhysteretic scalar that depends on $|\mathbf{H}|$.

Figure 5 shows the geometry. The shape of the magnetic slab has not been optimized, but for illustration purposes we have chosen a shape that concentrates the magnetic field in the gap near the bead. Symmetry has been used at $y=0$, and antisymmetry in the magnetic potential has been used at $x=0$, so that the entire geometric structure of the magnetic field is obtained by including the mirror images at $x=0$ and $y=0$. The boundary conditions are specified in the figure. It is also shown how the geometry depends on the dimensionless horizontal distance between the bead and the magnetic slab, $\varepsilon$, the dimensionless height of the bead center above the slab center, $\beta$, and the dimensionless difference between the slab thickness and the bead radius, $\delta$. A change in $\varepsilon$ corresponds to a translation of the magnetic slab, the geometry of the slab remains constant.

We treat both pieces of magnetic material as nonlinear and have used the relation $\mathbf{M}=M_{s} \tanh \left(\frac{\chi_{0}|\mathbf{H}|}{M_{s}}\right) \frac{\mathbf{H}}{|\mathbf{H}|}$, such that $\mu_{r}(|\mathbf{H}|)=1+\frac{M_{s}}{|\mathbf{H}|} \tanh \left(\frac{\chi_{0}|\mathbf{H}|}{M_{s}}\right)$, where $\mathbf{M}$ is the magnetization field, $M_{s}$ is the saturation magnetization, and $\chi_{0}$ is the initial susceptibility of the material. For the bead, we have used

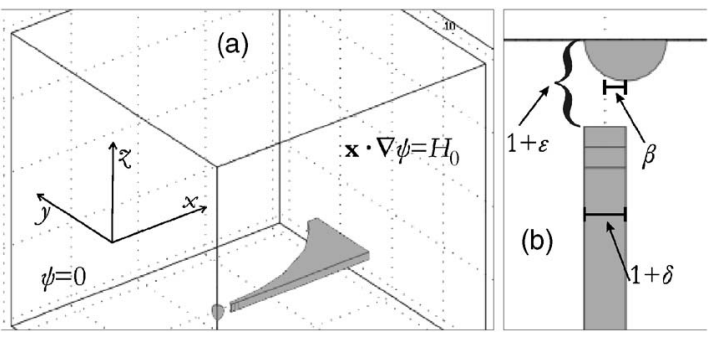

FIG. 5. Definitions of the geometry and boundary conditions for the magnetic calculations. (a) The three-dimensional calculation geometry. The gray region is the magnetic slab, and the bead is the quarter sphere. The coordinate system is defined on the figure, and the origin is chosen such that the center of the bead is at $(0,0,0)$, when $\beta=0$. The magnetic slab has length ( $x$ direction) 20 and maximum width ( $y$ direction) 10. The calculation domain is $40 \times 40 \times 40$. On the boundary where $x=x_{\max }$, the boundary condition is the applied homogenous magnetic field $H_{0}$, and at $x=0$, we have $\psi$ $=0$. On all other boundaries, the flux of the magnetic field is $\mathbf{n} \cdot \nabla \psi=0$. Symmetry considerations have been used, such that only one quarter of the entire magnetic structure (see Fig. 1) is included in the calculation domain. (b) A close-up of the region near the bead in the plane $y=0$. It has been rotated $90^{\circ}$ relative to (a). The geometric parameters $\varepsilon, \beta$, and $\delta$ are defined.

typical values $\chi_{0}=1.485$ and $M_{s}=39950 \mathrm{~A} / \mathrm{m},{ }^{23}$ and for the magnetic material, we have used the approximate parameters of electroplated permalloy, $\chi_{0}=1000$, and $M_{s}=8.8 \cdot 10^{5} \mathrm{~A} / \mathrm{m}$. For these calculations, we have used $H_{0}=50 \mathrm{kA} / \mathrm{m}$, which is easily generated by a table top electromagnet and has proven to be sufficient to saturate the bead as well as the magnetic slab.

The standard mesh was created choosing the "Normal mesh" under Comsol's mesh parameters and setting the mesh on boundaries on the surface of the bead to have "Maximum element size" 0.2 and "Element growth rate" 1.1. These steps gave a mesh with approximately 24000 elements. The fine mesh (used for the mesh investigation below) was produced by refining the standard mesh. This step resulted in a mesh with approximately 90000 elements.

We calculated the magnetic force on the bead by integration of the Maxwell stress tensor. We used the built-in Comsol Multiphysics Electromagnetics module routine ${ }^{24}$ to obtain the magnetic force by surface integration of ${ }^{22}$

$$
\mathbf{F}=\int_{\partial \Omega} \mathbf{n} \cdot\left(\mathbf{B H}-\frac{1}{2} \mu_{0} \mathbf{I}|\mathbf{H}|^{2}\right) d S,
$$

where $\mathbf{I}$ is the unit tensor, and $\partial \Omega$ is the outside surface of the quarter bead that is included in the calculation geometry. On the surfaces lying inside the bead, which are on the calculation domain boundaries, we did not use the built-in routine, but instead we used a postprocessing integration routine.

In the numerics we calculate the dimensional force using Eq. (15), and then we multiply by four to account for the whole sphere, and finally we normalize the force by the expected magnetic force given by Eq. (13).

\section{Numerical results for the fluid flow}

We now characterize the response of the fluid flow to changes in the geometric parameters $\varepsilon$, which is the dimensionless difference between the channel radius and the bead 

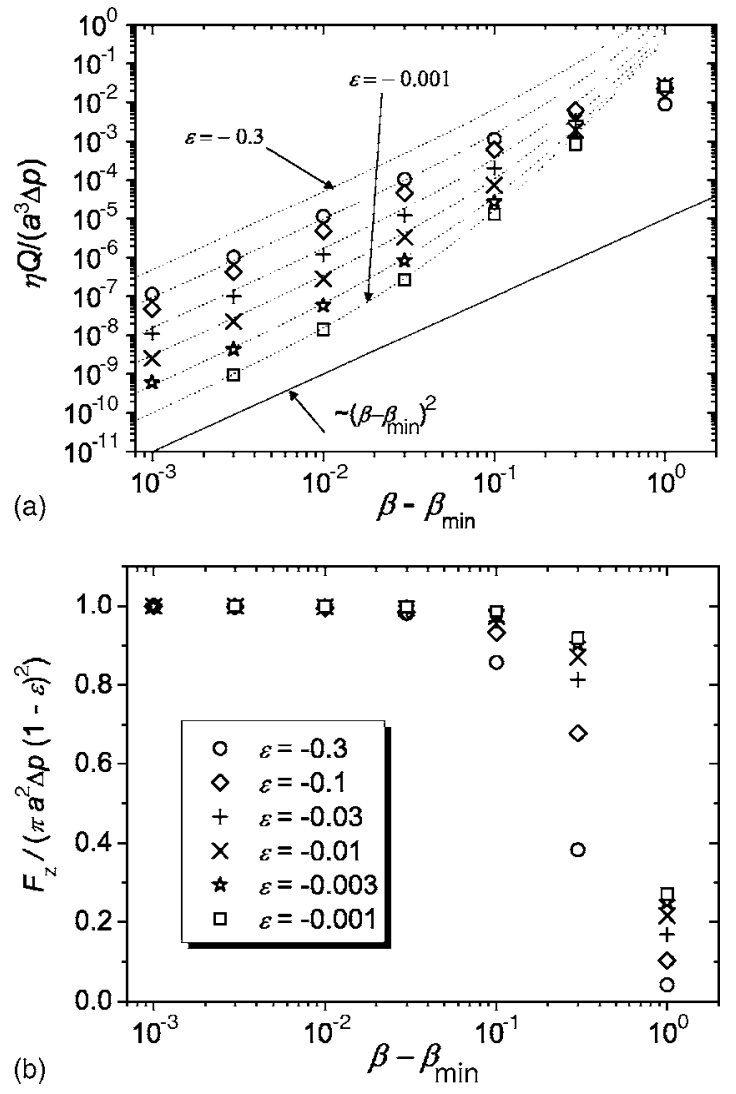

FIG. 6. Numerical results for the straight tube in the case where $\varepsilon<0$. (a) The hydrodynamic resistances for different values of $\varepsilon$ as a function of $\beta$. The dotted lines are the analytical results given by Eq. (10). (b) The fluid force in the $z$ direction on the magnetic bead for the same values of $\varepsilon$ as a function of $\beta$. The legend in (b) also applies to (a).

radius, and $\beta$, which is the dimensionless height of the center of the bead above the channel outlet. Figure 6 shows the results of calculations, where $\varepsilon<0$. In the theory section we showed that in this case there is a lower limit on $\beta$, $\beta>\beta_{\min }=\sqrt{-2 \varepsilon}$, such that the bead is always above the outlet of the cylindrical tube. Therefore the plots are made relative to $\beta_{\text {min }}$.

First we consider the hydrodynamic resistance, which is reported in Fig. 6(a). The symbols are the numerical data, and the dotted lines are the analytical result given by Eq. (10). It is seen that the analytical and numerical results agree well for small values of $\beta$ and $|\varepsilon|$; for example, for $\varepsilon=-0.01$ the analytical result is in good agreement with the numerical calculation for $\beta-\beta_{\min } \leq 0.1$.

For larger values of $|\varepsilon|$ the analytic expressions overestimate the flow rate by a factor of approximately 10 or more. For $\varepsilon=-0.3$, Eq. (8) fails to describe the conditions in the gap correctly, since $1-0.3=0.7 \approx \sin \left(45^{\circ}\right)$, and thus the gap on top of the channel between the bead and the opening of the channel at $x<0$ (refer to Fig. 3) dominates over the gap between the bead and the channel walls, and the gap on top of the channel is not treated by our calculations.

For $\beta-\beta_{\min } \geq 0.3$, the analytic result completely fails to describe the numerical data. This result is simply because the bead is now so far removed from the outlet that the hydro-
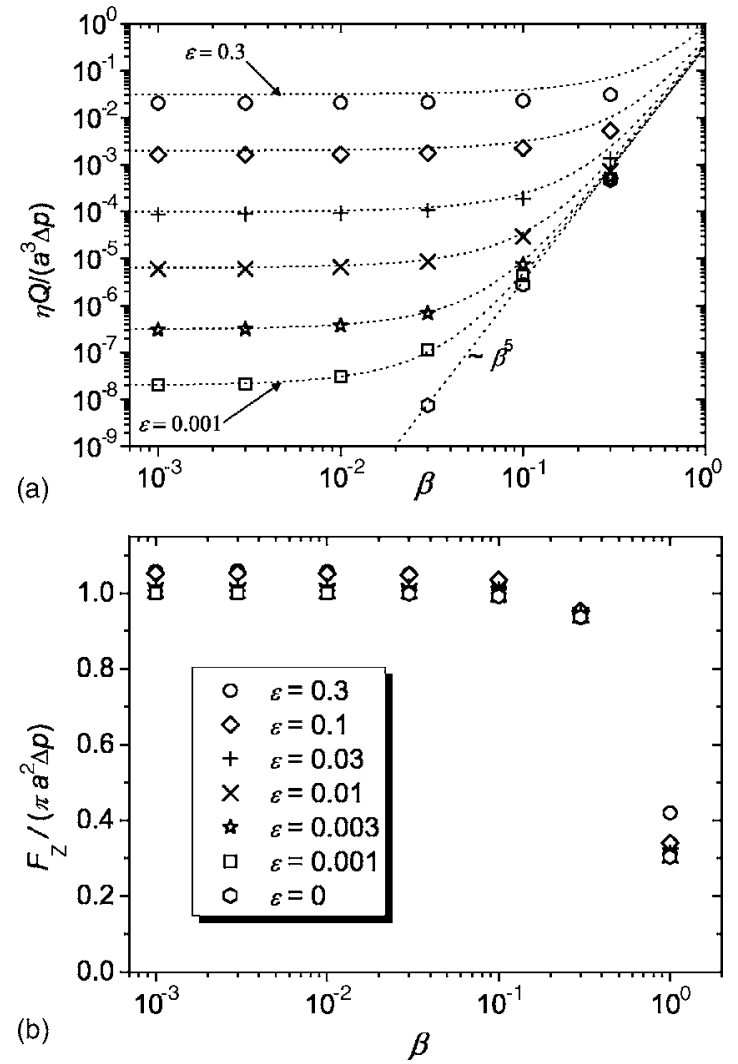

FIG. 7. Numerical results for the straight tube in the case where $\varepsilon \geq 0$. (a) The hydrodynamic resistances for different values of $\varepsilon$ as a function of $\beta$. The dotted lines are the analytical results given by Eq. (10). (b) The fluid force in the $z$ direction on the magnetic bead for the same values of $\varepsilon$ as a function of $\beta$. The legend in (b) also applies to (a).

dynamic resistance is no longer dominated by the presence of the bead, and without a narrow gap, the lubrication analysis is no longer valid.

For $|\varepsilon| \lesssim 0.1$ and $\beta \lesssim 0.1$, the lubrication analysis is in very good agreement with the numerical data, and it is seen that $\eta Q /\left(a^{3} \Delta p\right) \propto\left(\beta-\beta_{\text {min }}\right)^{2}$ as $\beta \rightarrow \beta_{\text {min }}$. This agrees with the result from the theory section.

We next consider the total fluid force on the bead and report the results in Fig. 6(b) relative to the analytically calculated force. The analytical result agrees very well for $\beta \leq 0.1$. This shows that the entire pressure drop is indeed across the gap, and that the pressure force dominates over the viscous force as expected.

Now we treat the case $\varepsilon \geq 0$. The hydrodynamic resistance is given in Fig. 7(a). The markers are the numerical data, and the dotted lines are the analytical result given by Eq. (10). Again, the analytical and numerical results agree well for all data except where $\varepsilon=0.3$. When the gap becomes large, the hydrodynamic resistance of the cylindrical tube becomes increasingly important, and thus lubrication analysis will overestimate the flow rate. When $\varepsilon$ is finite and $\beta$ is small, we see that the hydrodynamic resistance goes towards a constant as expected from the theory section. This is due to the fact that when $\beta$ is much smaller than $\varepsilon$, then the geometry of the gap is dominated by $\varepsilon$ rather than $\beta$. For $\varepsilon=0$, and for values of $\beta$, where the geometry of the gap is dominated by $\beta$ rather than $\varepsilon, \eta Q /\left(a^{3} \Delta p\right) \propto \beta^{5}$. 

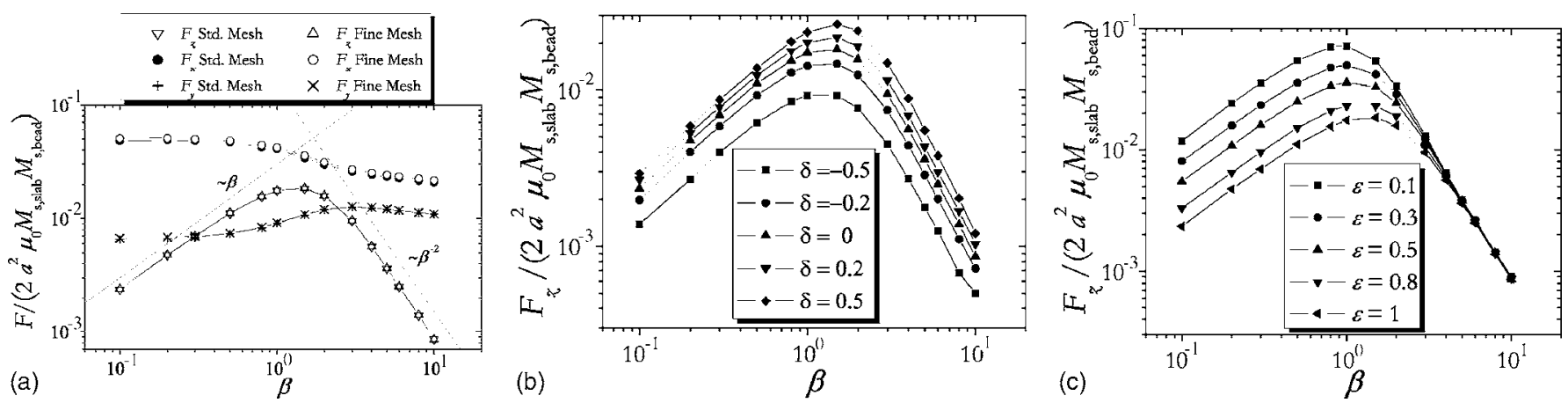

FIG. 8. Numerically calculated magnetic forces (absolute values) for various values of the parameters. All forces have been normalized by the value expected in Eq. (13). The force calculations have been done for the magnetic geometry shown in Fig. 5 and Fig. 1(d), and any of the fluidic designs Figs. 1(a)-1(c) can be applied. (a) Magnetic force calculations with the standard mesh and a further refined mesh. $\varepsilon=1$ and $\delta=0$. It is indicated how the force changes with $\beta$. (b) The force in the $z$ direction as a function of $\beta$ for different values of $\delta, \varepsilon=1$. (c) The force in the $z$ direction as a function of $\beta$ for different values of $\varepsilon$; $\delta=0$.

Finally, we report the results for the forces in units of the analytical lubrication result in Fig. 7(b). For $\beta \leqq 0.1$, and $\varepsilon$ $\lesssim 0.1$ the numerical and analytical data agree very well. The discrepancy in the data for larger values of $\varepsilon$ is caused by the substantial fluid flow present even for $\beta=0$, which causes a significant viscous force.

\section{Numerical results for the magnetic force}

We calculate the obtainable magnetic forces by variations of the geometric parameters $\varepsilon, \beta$, and $\delta$ in Fig. 8. We compare force calculations with the standard mesh to calculations with the fine mesh in Fig. 8(a) and find that the standard mesh is sufficient for these calculations. The forces in the $x$ and $y$ directions are not really meaningful, since both include an unbalanced magnetic pressure that would still be present in all directions if the bead was moved a little in either direction.

We investigate the influence on the magnetic force in the $z$ direction of the thickness of the magnetic slab, described by $\delta$, in Fig. 8(b). We find that it is preferable to have a thick magnetic slab. However, there must be an upper limit where a thicker magnetic slab does not contribute with any more force. This limit is most likely related to $\varepsilon$. It is outside the scope of this paper to determine this limit.

Finally, we present the forces in the $z$ direction for different values of the distance between the magnetic slabs, which is determined by $\varepsilon$ in Fig. 8(c). We find that the width of the gap, $\varepsilon$, has little influence on the force outside the gap, i.e., when $\beta>1$. However, when the bead approaches the gap, it is seen that it is best to have a small gap ( $\varepsilon$ should be small).

From Fig. 8 we see that we cannot generate the force given by Eq. (13), but we can get close to one tenth of this force. In addition, Fig. 8(b) shows that more force could be gained by increasing $\delta$ further, and also a more powerful applied magnetic field might increase the force. In Fig. 8 we found good design rules for design of the magnetic slabs; they should be thick, probably at least twice the diameter of the bead, and the gap between the magnetic slabs should be as small as possible.

\section{DISCUSSION AND OUTLOOK}

In this paper, we have proposed a new design for a magnetically actuated valve that, in principle, may be reasonable for designs on the nanoscale, since control of the valve position is done externally. We have developed analytical expressions for the fluid forces and fluid flow through the valve at different conditions, and we have verified the results numerically. In addition, we have determined how the magnetic force depends on the same parameters, and from the results of the numerical calculations we have found design rules for optimum choices of the width of the gap, $\varepsilon$, and the thickness of the magnetic slabs, $\delta$.

With the designs we have modelled here and typical material parameters, we have shown that it is possible to reach a force which is approximately one tenth of what we would expect from the scaling argument in Eq. (13), and thus referring to Eq. (14), we find that it is possible to withstand back pressures of approximately $3 \mathrm{kPa}$. With further improvements it should be possible to get close to the $3 \mathrm{kPa}$ mentioned in Eq. (14).

The design in Fig. 1(a), where $\varepsilon>0$, has the property that if you have a tightly fitting tube with $|\varepsilon| \ll 1$, it is possible to have a change in hydrodynamic resistance over several orders of magnitude. The alternative design in Fig. 1(b) facilitates complete fluid blocking, and also the fluid forces can be reduced by choosing a more negative $\varepsilon$. However, the fitting and closing properties of the valve will then be diminished. The design in Fig. 1(c) clearly has the best properties for positioning the bead in the middle of the channel and flow blocking. The bead will automatically be guided into the opening of the tapering tube. Also, most commercially available magnetic beads have a considerable size distribution. Bead size distribution may be a problem with the two other designs, especially, if $|\varepsilon| \ll 1$ is desired, but the design in Fig. 1(c) overcomes this problem due to the tapering sidewalls. This design, however, poses a greater challenge in terms of microfabrication.

In all the calculations, we have assumed that the bead stays in the center of the fluid channel or between the magnetic slabs. Experimentally this may not be the case. The 
bead may have a tendency to go to the side allowing for a larger fluid flow. This consideration favors the use of the design in Fig. 1(c), which will diminish this potential problem.

As the characteristic length scale (the bead radius) is reduced, the fluid-blocking efficiency for all the designs is limited by Brownian motion, which will cause the bead to oscillate around the closing position. Again, if the bead is away from the center the result will be greater flow than expected from the calculations. The quantitative influence of Brownian motion on the results shown here is a subject of active investigation. However, it is straightforward to estimate a bead radius, where the valve design breaks down completely when Brownian motion dominates over the magnetic force (which is equal to the maximum pressure force that can be applied). We simply compare thermal energy to the energy/work required to move a bead a distance of one bead radius against the magnetic force [Eq. (13)],

$$
\frac{E^{\text {thermal }}}{E^{\text {mag }}}=\frac{k T}{2 \mu_{0} M_{s, \text { bead }} M_{s, \text { slab }} a^{3}},
$$

which at room temperature and for a $0.5 \mu \mathrm{m}$ radius Invitrogen MyOne bead considered above is $\approx 4 \cdot 10^{-8}$, showing that Brownian motion is unimportant at this bead size. However, this ratio becomes unity as the bead radius is reduced to $4 \mathrm{~nm}$ (assuming that all other material properties are the same. In reality the bead saturation magnetization will most likely increase by approximately one order of magnitude).

The above scaling argument shows that the valve will not break down due to Brownian motion, as long as the bead/particle radius is more than approximately $10-20 \mathrm{~nm}$. However, since we require that the fluid flow is completely dominated by the flow in the gap, we must require that the gap height is at least a few nanometers. Otherwise the assumption of a continuum fluid breaks down, and our calculations no longer apply. If we further assume that the bead diameter should be at least ten times the gap for the lubrication approximation to hold, then the bead must have a diameter of at least $40 \mathrm{~nm}$. Such bead diameters are well above the limit where the valve breaks down due to Brownian motion.

In all microsystems and even more in nanosystems, stiction and van der Waals forces play an important role, and should definitely be considered, when one wants to fabricate valves on the nanoscale. This applies for any valve design on this length scale. However, recently there has been substantial progress towards coatings that are nonstick on the nanoscale (see, for example, Ref. 25). Comparing the numerical values given in Ref. 25 to Eq. (13) shows that the magnetic force should be sufficient to overcome stiction for beads with diameters of 5 and $20 \mu \mathrm{m}$.

Finally, we note that we have demonstrated a valve design with magnetic forces that will withstand fluid pressures of $3 \mathrm{kPa}$ and that further optimization of the shape of the magnetic elements should increase this number. Also, magnetic particles with higher saturation magnetizations exist, and if those were used, higher forces and pressures could be reached. It is not unreasonable to expect an improvement by a factor of 5-10 on that account. We have argued that the continuum assumption breaks down before the valve is affected by Brownian motion. But still Brownian motion may play an important role in the performance of all of the valve designs but especially the one in Fig. 1(a). Stiction may be a problem, and it will most likely be the limiting factor for downscaling. As further progress is made with nonstick coatings, it will most likely be possible to fabricate magnetically actuated ball valves utilizing beads with diameters in the range of hundreds of nanometers or less.

\section{ACKNOWLEDGMENTS}

The Danish Technological Institute and the Copenhagen Graduate School for Nanoscience and Nanotechnology (C.O.N.T.) are acknowledged for financially supporting K.S.'s Ph.D. studies. We thank the Harvard NSEC for partial support of this research and Benny Davidovitch for helpful conversations.

${ }^{1}$ T. M. Squires and S. R. Quake, "Microfluidics: Fluid physics at the nanoliter scale," Rev. Mod. Phys. 77, 977 (2005).

${ }^{2}$ H. A. Stone, A. D. Stroock, and A. Ajdari, "Engineering flows in small devices: Microfluidics toward a lab-on-a-chip," Annu. Rev. Fluid Mech. 36, 381 (2004).

${ }^{3}$ Microsystem Engineering of Lab-on-a-Chip Devices, edited by O. Geschke, H. Klank, and P. Telleman (Wiley-VCH, Weinheim, 2004).

${ }^{4}$ J. Knight, "Honey, I shrunk the lab," Nature (London) 418, 474 (2005).

${ }^{5}$ B. G. Stubbe, S. C. De Smedt, and J. Demeester, "Programmed polymeric devices for pulsed drug delivery," Pharm. Res. 21, 1732 (2004).

${ }^{6}$ A. Grayson, R. Shawgo, A. Johnson, N. Flynn, Y. Li, M. Cima, and R. Langer, "A biomems review: Mems technology for physiologically integrated devices," Proc. IEEE 92, 6 (2004).

${ }^{7}$ P. Gravesen, J. Branebjerg, and O. S. Jensen, "Microfluidics: A review," J. Micromech. Microeng. 3, 168 (1993).

${ }^{8}$ P. K. Yuen, L. J. Kricka, and P. Wilding, "Semi-disposable microvalves for use with microfabricated devices or microchips," J. Micromech. Microeng. 10, 401 (2000).

${ }^{9}$ D. J. Laser and J. G. Santiago, "A review of micropumps," J. Micromech. Microeng. 14, R35 (2004).

${ }^{10}$ D. Accoto, M. Carrozza, and P. Dario, "Modelling of micropumps using unimorph piezoelectric actuator and ball valves," J. Micromech. Microeng. 10, 277 (2000).

${ }^{11}$ M. Carrozza, N. Croce, B. Magnani, and P. Dario, "A piezoelectric-driven stereolithography-fabricated micropump," J. Micromech. Microeng. 5, 177 (1995).

${ }^{12}$ D. B. Weibel, M. Kruithof, S. Potenta, S. K. Sia, A. Lee, and G. M. Whitesides, "Torque-actuated valves for microfluidics," Anal. Chem. 77, 4726 (2005).

${ }^{13}$ J. M. K. Ng, I. Gitlin, A. D. Stroock, and G. M. Whitesides, "Components for integrated poly(dimethylsiloxane) microfluidic systems," Electrophoresis 23, 3461 (2002).

${ }^{14}$ B. J. Kirby, D. S. Reichmuth, R. F. Renzi, T. J. Shepodd, and B. J. Wiedenman, "Microfluidic routing of aqueous and organic flows at high pressures: fabrication and characterization of integrated polymer microvalve elements," Lab Chip 5, 184 (2005).

${ }^{15}$ B. J. Kirby, T. J. Shepodd, and E. F. Hasselbrink, "Voltage-addressable on/off microvalves for high-pressure microchip separations," J. Chromatogr., A 979147 (2002).

${ }^{16}$ E. F. J. Hasselbrink, T. J. Shepodd, and J. E. Rehm, "High-pressure microfluidic control in lab-on-a-chip devices using mobile polymer monoliths," Anal. Chem. 74, 4913 (2002).

${ }^{17}$ S. D. Solares, M. Blanco, and W. A. Goddard III, "Design of a nanomechanical fluid control valve based on functionalized silicon cantilevers: Coupling molecular mechanics with classical engineering design," Nanotechnology 15, 1405 (2004).

${ }^{18}$ T. D. Nguyen, H.-R. Tseng, P. C. Celestre, A. H. Flood, Y. Liu, J. F. Stoddart, and J. I. Zink, "A reversible molecular valve," Proc. Natl. Acad. Sci. U.S.A. 102, $10029(2005)$. 
${ }^{19}$ See www.magneticmicrosphere.com for suppliers of magnetic beads and particles.

${ }^{20} \mathrm{H}$. A. Stone, "On lubrication flows in geometries with zero local curvature," Chem. Eng. Sci. 60, 4838 (2005).

${ }^{21}$ G. K. Batchelor, An Introduction to Fluid Dynamics (Cambridge University Press, Cambridge, 1967).

${ }^{22}$ A. Engel and R. Friedrichs, "On the electromagnetic force on a polarizable body," Am. J. Phys. 70, 428 (2002).
${ }^{23}$ G. Fonnum, C. Johansson, A. Molteberg, S. Mørup, and E. Aksnes, "Characterization of dynabeads by magnetization measurements and mössbauer spectroscopy," J. Magn. Magn. Mater. 293, 41 (2005).

${ }^{24} \mathrm{Comsol}$ support and Comsol Multiphysics documentation, www.comsol.com

${ }^{25}$ K. Gjerde, J. Kjelstrup-Hansen, C. H. Clausen, K. B. K. Teo, W. I. Milne, H Rubahn, and P. Boggild, "Carbon nanotube forests: A nonstick workbench for nanomanipulation," Nanotechnology 17, 4917 (2006). 